\title{
Environmental Effects on Galaxy Luminosity Functions in Clusters
}

\author{
Florence Durret ${ }^{1,2}$, Christophe Adami $^{3}$ and Tatiana F. Laganá ${ }^{4}$ \\ ${ }^{1}$ UPMC Université Paris 06, UMR 7095, Institut d'Astrophysique de Paris, 98bis Bd Arago, \\ F-75014, Paris, France, email: durret@iap.fr \\ ${ }^{2}$ CNRS, UMR 7095, Institut d'Astrophysique de Paris, F-75014 Paris, France \\ ${ }^{3}$ LAM, Pôle de l'Etoile Site de Château-Gombert, 38 rue Frédéric Joliot-Curie, \\ 13388 Marseille Cedex 13, France \\ ${ }^{4}$ IAG, USP, R. do Matão 1226, 05508-090, São Paulo/SP, Brazil
}

\begin{abstract}
The formation and evolution of galaxies is strongly influenced by environment, particularly in clusters, where galaxy luminosity functions vary in shape with the dynamical state of the cluster (relaxed or in various stages of merging), with the photometric band considered and with the position in the cluster. We present here results concerning the optical GLFs in several relaxed and merging clusters.
\end{abstract}

Keywords. galaxies: clusters: individual (Coma, A85, A222/223, A496, A780, A1758), galaxies: luminosity function

\section{Introduction}

Galaxy luminosity functions (GLFs) in clusters are known to be under the influence of their environment, particularly their faint end slopes. For example, they tend to be flatter in the inner regions, where accretion of dwarf galaxies by larger ones is more frequent, and steeper in the outskirts. They also tend to be steeper in the blue, implying that dwarf galaxies are rather blue. Star formation is also a function of environment: numerical simulations have shown that the star formation rate can both be enhanced by shocks due to cluster mergers, and quenched if mergers strip the galaxies from their gas. We illustrate these properties by showing GLFs in several relaxed and merging clusters. Cluster mergers also have a strong effect on the cluster X-ray emitting gas, but due to lack of space this will not be discussed here.

GLFs (as a function of absolute magnitude $M$ ) are usually fit by a Schechter function:

$$
S(M)=0.4 \ln 10 \phi^{*} y^{\alpha+1} e^{-y}
$$

where $\alpha$ is the faint end slope, $\phi^{*}$ a normalization factor and $y=10^{0.4\left(M^{*}-M\right)}$. In some cases, a Schechter function is not sufficient to fit the GLF over the entire magnitude range.

\section{Relaxed clusters}

The global GLF of A780 (Hydra A, redshift $\mathrm{z}=0.0539$ ) has a composite shape, with a rather flat faint end slope $\alpha=-1.09 \pm 0.05$ (see Fig. 1 and Durret et al. 2009) comparable to that found for field galaxies (Ilbert et al. 2005). This agrees with the fact that this cluster appears totally relaxed, with no obvious substructures or links with its surroundings, such as large scale filaments or galaxies distributed along preferential directions. 
On the other hand, the global faint end slope is steeper in A496 (redshift $\mathrm{z}=0.033$ ): $\alpha=-1.68 \pm 0.35$ in $u^{*}$ and $\alpha=-1.52$ to -1.62 in the other bands $\left(g^{\prime}, r^{\prime}, i^{\prime}\right)$. If we divide A496 into 16 square regions (Fig. 1), the GLF appears flatter in the central squares than in the outer ones. If we reassemble these zones into three regions, the result is that in the central zone (the blue square) $\alpha=-1.60 \pm 0.27$ in $u^{*}$ and $\alpha=-1.40 \pm 0.08$ in the other bands, while in the south (red rectangle) $\alpha=-1.80$ in all bands, and in the east-northwest zone (marked in green) we have $\alpha=-1.88$ in $u^{*}$ and $\alpha=-1.4$ to -1.6 in the other bands. Differences in the faint end slope are therefore found from one region to another. The fact that $\alpha$ is steeper in the outskirts may be related to infall (or creation) of dwarf galaxies along a filament of galaxies detected at very large scales (see Boué et al. 2008a, Fig. 10).
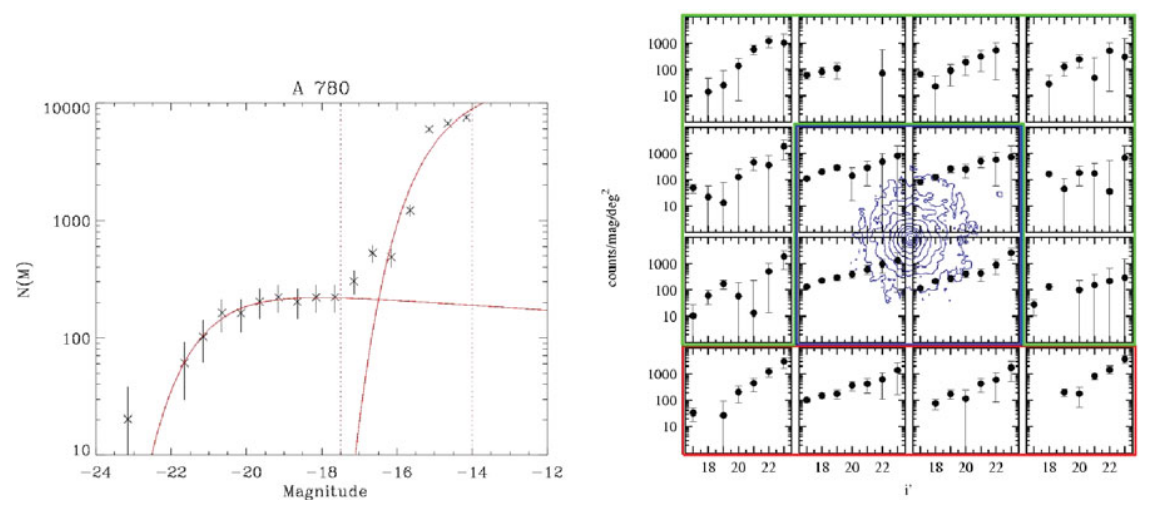

Figure 1. Left: GLF of A780 in the R band with fit by two Schechter functions superimposed. Right: $r^{\prime}$ band GLFs in 16 regions of A496 with X-ray contours in blue.

\section{Non-relaxed clusters}

The Coma cluster (redshift $\mathrm{z}=0.023$ ), once believed to be the prototype of a relaxed cluster, has been shown since the 1990's to be very perturbed. Its GLFs plotted in 20 rectangular regions show much flatter faint end slopes south of the red line than north of it (see Fig. 2). The mean values for the north and south regions are $\alpha=-1.74 \pm 0.02$

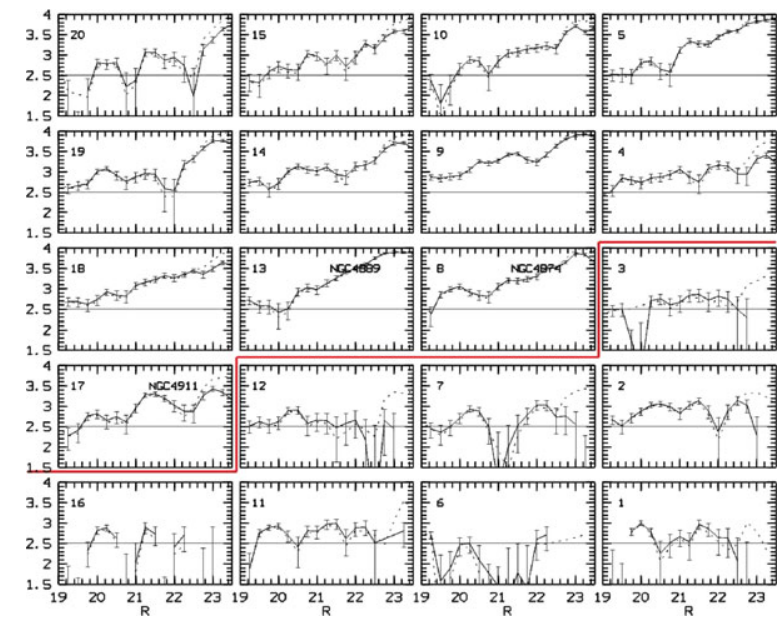

Figure 2. GLF in various regions of the Coma cluster in the $\mathrm{R}$ band. 
and $\alpha=-1.28 \pm 0.05$ respectively ( $\mathrm{R}$ band). Such a flat slope in the south region is very difficult to explain, since infall indeed seems to be coming from the south, where $\alpha$ is expected to be steeper. A full discussion can be found e.g. in Adami et al. (2007).

A85 (redshift $\mathrm{z}=0.055$ ) has been undergoing several mergers in the last few Gyrs and is still under the effect of a merger, seen as groups falling on to the main cluster and detected both as an X-ray filament (Durret et al. 2003, 2005) and as a zone where galaxies tend to show a higher star formation rate, though surprisingly no differences are found for the faint end slopes in various regions (Boué et al. 2008b). On the other hand, as for other clusters, the faint end slope is possibly steeper in the blue, but the error bar is large: $\alpha=-1.7 \pm 0.5$ in $u^{*}$ and $\alpha=-1.5 \pm 0.1$ in the other bands.

\section{Cluster pairs}

We have recently undertaken a survey of cluster pairs, where merging effects are expected to be even more important, based on similar data as above. The pairs considered here are somewhat more distant because we required that the two clusters forming each pair fit in an XMM-Newton field (diameter $<30$ arcmin).

From the properties of the X-ray gas, we can derive that the A222/223 cluster pair (redshift $\mathrm{z}=0.21$ ) is made of two quite different clusters, A222 being rather relaxed while A223 has just been crossed by a small subcluster (Durret et al. 2010). The GLFs of A222 and A223 (see Fig. 3) are very similar in the $r^{\prime}$ band, with $\alpha \sim-1.3$. The slope is the same for A223 in the $g^{\prime}$ band, while it is much flatter in A222 in $g^{\prime}: \alpha \sim-0.6$ to -0.9 , depending on the background subtraction. An excess of galaxies over a Schechter function is observed at bright magnitudes, and several wiggles are detected in all GLFs.

The A1758N/S pair (redshift $\mathrm{z}=0.279$ ) is quite comparable to A222/223, though it is even more perturbed, since each cluster is in fact double. The faint end slopes are $\alpha \sim-0.9$ in both bands in A1758N, while for A1758S we find $\alpha \sim-0.6$ or -1.1 in the $g^{\prime}$ band and $\alpha \sim-1.1$ in the $r^{\prime}$ band (see Fig. 4 and Durret et al. 2011), depending
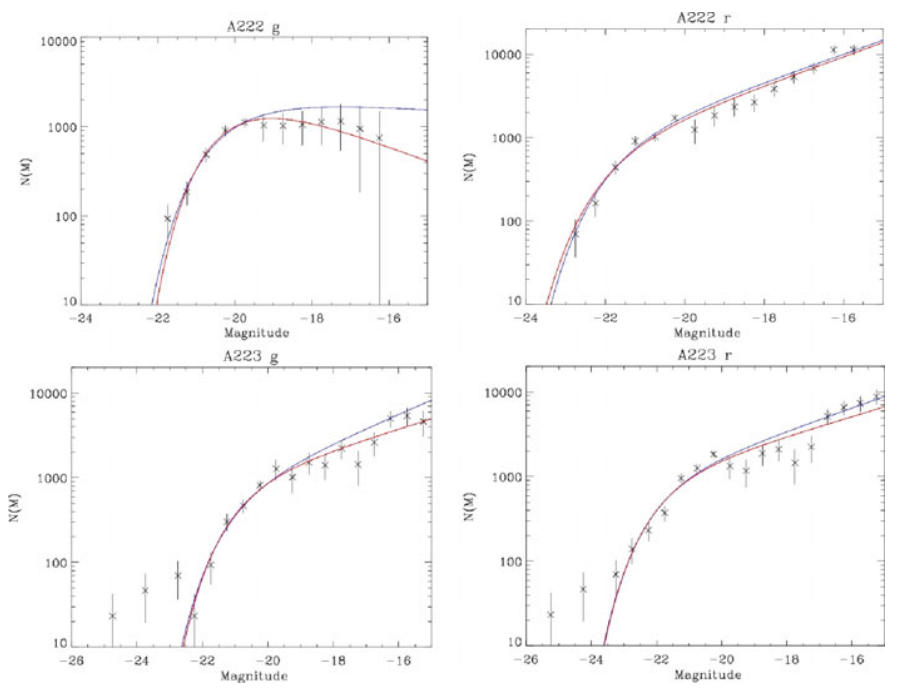

Figure 3. GLFs of the A222 (top) and A223 (bottom) clusters in the $g^{\prime}$ (left) and $r^{\prime}$ (right) bands. The red and blue lines correspond to the best Schechter fits on GLFs estimated with two different background subtractions. 

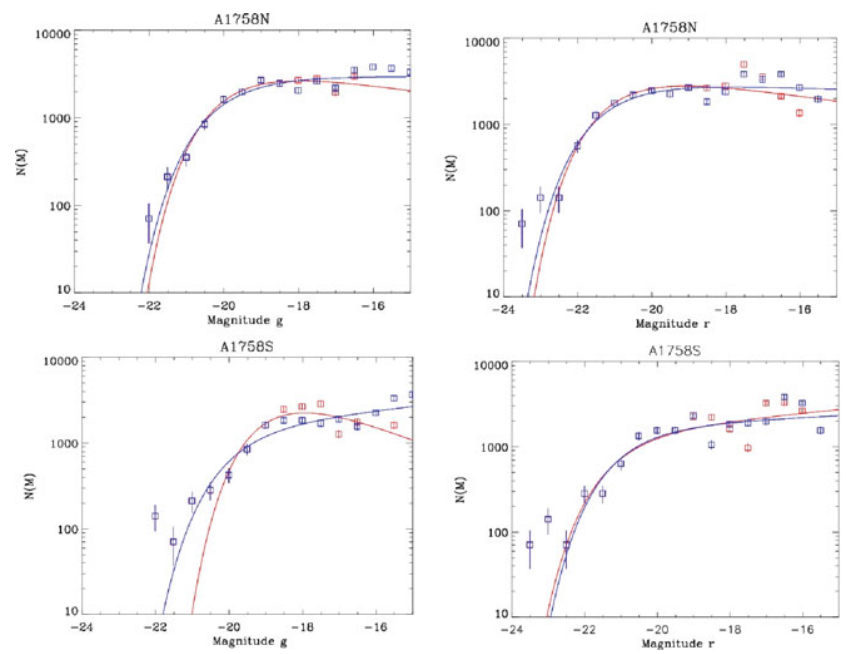

Figure 4. GLFs of the A1758N (top) and A1758S (bottom) clusters in the $g^{\prime}$ (left) and $r^{\prime}$ (right) bands. The red and blue lines correspond to the best Schechter fits on GLFs estimated by two different methods (see text).

on the determination method (statistical background subtraction or selection based on a colour-magnitude diagram).

\section{Discussion}

The above results illustrate several disturbing facts. First, though the study of GLFs can give insight on dynamical and merging processes, the correlation is not perfect. In particular, though steep faint end slopes may be linked to infall from the surrounding medium, clusters showing obvious mergers do not always have steep GLF slopes. Second, error bars on the faint end slopes are probably underestimated and GLFs should be derived at least with two different methods, for example one based on a statistical background subtraction and the other on the selection of cluster galaxies based on the colour-magnitude relation. The hypothesis of spherical symmetry should be avoided in all cases, since infall of material on to clusters is not isotropic.

\section{Acknowledgements}

Due to lack of space, it was not possible to include as co-authors all our collaborators. May they be thanked for their contributions.

\section{References}

Adami C., Durret F., Mazure A. et al. 2007, A\&A 462, 411

Boué G., Adami C., Durret F., Mamon G., \& Cayatte V. 2008a, A $8 A$ 479, 335

Boué G., Durret F., Adami C., Mamon G., Ilbert O., \& Cayatte V. 2008b, A\& A 489, 11

Durret F., Gerbal. D., Lobo C., \& Pichon C. 1999, A $\& A$ 343, 760

Durret F., Lima Neto G. B., Forman W., \& Churazov E. 2003, A\&A 403, L22

Durret F., Lima Neto G. B., \& Forman W. 2005, A\&A 432, 809

Durret F., Slezak E., \& Adami C. 2009, A\&A 506, 637

Durret F., Laganá T. F., Adami C., \& Bertin E. 2010, A\& $A$ 517, 94

Durret F., Laganá T. F., \& Haider M. 2011, A\&A submitted

Ilbert O., Tresse L., Zucca E., et al. 2005, A $\mathscr{E} A$ 439, 863 